\title{
Magnification Effect of Foveal Avascular Zone Measurement Using Optical Coherence Tomography Angiography
}

\author{
Mika Suda $^{a}$ Yuji Yoshikawa $^{b}$ Gaku Terauchic $^{c}$ Soiti Matsumoto ${ }^{c}$ \\ Takuhei Shoji $^{b}$ Kei Shinoda ${ }^{b}$ Atsushi Mizotac Yoshiharu Kobayashi ${ }^{a}$ \\ a Department of Orthoptics, Teikyo University Faculty of Medical Technology, Tokyo, Japan; \\ ${ }^{b}$ Department of Ophthalmology, Saitama Medical University, Saitama, Japan; ' Department \\ of Ophthalmology, Teikyo University School of Medicine, Tokyo, Japan
}

\begin{abstract}
What Is It about?
The area of the foveal avascular zone (FAZ) can be measured using optical coherence tomography angiography. The magnification effect at the time of fundus imaging can influence the FAZ area, as it can be over- or underestimated without correction of the effect. We examined the magnification effect on the FAZ measurement of healthy subjects and found that in the eyes with an axial length $\geq 26 \mathrm{~mm}$, the FAZ area after correction was significantly larger than that before correction $(p<0.01)$. Therefore, the magnification effect needs to be considered when evaluating the FAZ area.
\end{abstract}

\section{Keywords}

Foveal avascular zone · Magnification effect · Optical coherence tomography angiography · Myopia $\cdot$ Axial length

\begin{abstract}
Purpose: The aim of this study was to evaluate the foveal avascular zone (FAZ) of healthy subjects and examine the magnification effect. Methods: A total of 33 healthy volunteers were enrolled and all subjects were eligible for analysis. Optical coherence tomography angiography (OCTA) examination scanned $3 \times 3 \mathrm{~mm}$ of the macular area. The FAZ area was measured on the superficial OCTA en face image with and without correction by axial length. The relationship between changes in the FAZ area after correction with the axial length was examined. Results: The mean age was $21.9 \pm 0.6$ years. The mean axial length was $24.87 \pm 1.17 \mathrm{~mm}$ and mean spherical equivalent (SE) value was $-3.64 \pm 2.83$ diopters (D). The FAZ area was $0.26 \pm$ $0.10 \mathrm{~mm}^{2}$ before the axial length correction and $0.27 \pm 0.10 \mathrm{~mm}^{2}$ after the correction. In the eyes that had an axial length longer than or equal to $26 \mathrm{~mm}$ or SE less than or equal to $-6 \mathrm{D}$, the FAZ area after correction was significantly larger than that before correction $(p<0.01)$.
\end{abstract}


The change of FAZ area after correction with axial length was significantly correlated with the axial length $\left(R^{2}=0.88, p<0.01\right)$ or SE value $\left(R^{2}=0.55, p<0.01\right)$. Conclusion: FAZ areas were comparable to previous reports. In high myopic cases, the magnification effect needs to be considered when evaluating the FAZ area.

(c) 2020 The Author(s)

Published by S. Karger AG, Basel

\section{Introduction}

The foveal avascular zone (FAZ) is the avascular area of the central macula. It demonstrates large individual variations in size and shape [1]. The FAZ has been studied as an indicator of vasculature change in the retina [2]. Previous studies investigating the FAZ used fluorescein angiography [3], but a number of recent reports using optical coherence tomography (OCT) angiography (OCTA) have been published [4-12]. OCTA allows non-invasive evaluation of the retinal and choroidal circulation without the need for dye injection [5].

Previous studies have reported enlarged FAZ areas in diabetic retinopathy [6], retinal vascular obstruction [7], and macular hole [8]. A decreased FAZ area has been shown in epiretinal membrane [9] and premature retinopathy [13]. These studies have demonstrated that quantification of FAZ using OCTA may provide useful information for the diagnosis of ocular diseases.

While a scan size of $3 \times 3$ or $6 \times 6 \mathrm{~mm}$ is widely used, individual differences in axial length affect the absolute dimensions of the scan. Linderman et al. [14] reported that if this magnification effect is not corrected, measurements of the FAZ will be inaccurate.

Myopia is an important public health concern, with a rapid increase in prevalence in recent decades worldwide [15]. Although the FAZ area value is clinically useful for myopia diagnosis, few reports [16] have considered magnification effect at the time of fundus imaging on the FAZ area. Correction of axial length or spherical equivalent (SE) with these measurements has rarely been performed. In such a case, the FAZ area can be over- or underestimated. Linderman et al. [14] reported that not correcting for axial length introduced errors of up to $31 \%$ in FAZ area. Therefore, it is necessary to investigate the magnification effect and its influence on the FAZ area measurement for each OCTA device. We evaluated the FAZ area in healthy control subjects and investigated the magnification effect for FAZ area measurement.

\section{Methods}

\section{Study Design}

We investigated the magnification effect for FAZ area measurement using spectraldomain OCTA (SD-OCTA, RS3000 Advance, Nidek Co. Ltd, Japan) and evaluated the relationship between FAZ area and axial length or SE. This prospective, cross-sectional, and observational study was approved by the Ethics Committee of Teikyo University School of Medicine (study ID No.15-228). All studies adhered to the tenets of the Declaration of Helsinki and written informed consent was obtained from each participant before any study examination was performed.

\section{Study Subjects and Examinations}

Thirty-three healthy students of the Department of Orthoptics, Teikyo University Faculty of Medical Technology were recruited between April 2016 and March 2017. All participants underwent axial length measurement (Optical Biometer OA-1000, Tomey Corp., Japan) and SD-OCT and OCTA examination (RS3000 Advance, Nidek Co. Ltd, Japan). We included healthy 
Fig. 1. Representative cases for OCTA macula scan before and after axial length correction. For the non-high myopic eye (i.e., axial length shorter than $26 \mathrm{~mm}$ ), there were no significant magnification effects. For the high myopic eye (i.e., axial length longer than or equal to $26 \mathrm{~mm}$ ), the true macula scan area after axial length correction was larger than the default scan area $(3 \times 3 \mathrm{~mm})$. If we measured the FAZ area with the default setting, the scan area was decreased to $3 \times 3 \mathrm{~mm}$ and resulted in a decreased, incorrect FAZ area.

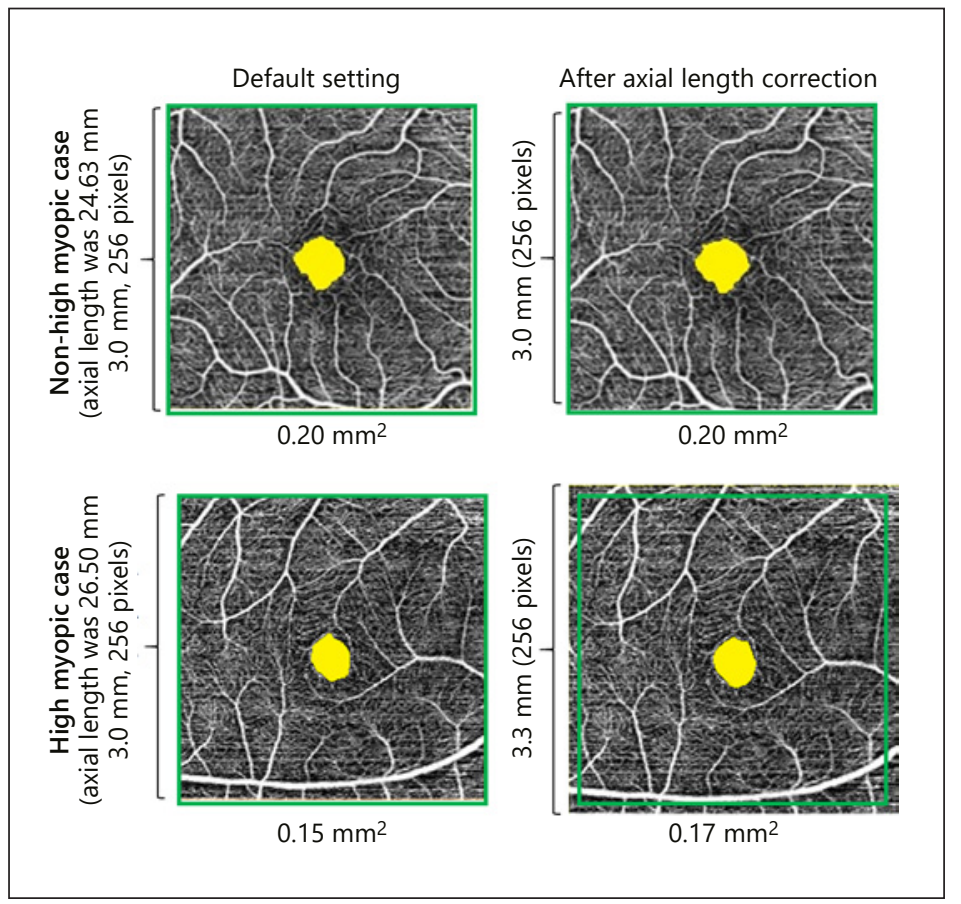

individuals with no ocular disease except refractive error, no history of ophthalmological or medical problems (e.g., hypertension, diabetes, cardiovascular disease), a normal fundus examination, and a normal structural OCT of the macula. We evaluated the image of the right eye from each participant.

\section{Optical Coherence Tomography Angiography}

A $3 \times 3$-mm volume OCTA scan centered on the fovea was performed twice on the same day. The OCTA en face image was obtained using a central wavelength of $880 \mathrm{~nm}$ and an A-scan rate of 53,000 scans/s. The axial and transverse image resolution in the imaged tissue was 7 and $20 \mu \mathrm{m}$, respectively. The OCTA software was used to acquire a $3 \times 3 \times 2$-mm image, with each cube consisting of 256 B-scans. Angiography signals were processed using both phase/Doppler shift and amplitude variation, also known as complex OCTA signal difference analysis angiography (CODAA). The automated segmentation defines the en face superficial retinal layer as extending from $8 \mu \mathrm{m}$ below the internal limiting membrane to the inner boundary of the inner nuclear layer [17].

\section{FAZ Measurement and Correction with Axial Length}

We obtained the superficial retinal en face image with built-in segmentation software. Whole edge points were manually connected to each other along the borderline of the identifiable capillary network in the macula area and calculated area $\left(\mathrm{mm}^{2}\right)$ with built-in software. The details of how magnification correction was performed were not disclosed. Moreover, the correction of FAZ area with ocular axial length was performed with built-in software (Fig. 1).

\section{Statistical Analysis}

All data are shown as the mean \pm standard deviation (SD) and mean (95\% confidence interval). The reproducibility of the FAZ area between two scans was evaluated with the coefficient of variation (CV) and the inter-class correlation coefficient (ICC). 
Table 1. Clinical characteristics $(n=33)$
Age, years
$21.9 \pm 0.6$
Female/male, $n$
$11 / 22$
Axial length, $\mathrm{mm}$
$24.87 \pm 1.17$
SE, D

Data are shown as the mean \pm SD. SE, spherical equivalent.

Table 2. Area of the FAZ

\begin{tabular}{|c|c|c|c|c|c|}
\hline & \multirow[t]{2}{*}{ Overall } & \multicolumn{2}{|c|}{ Axial length } & \multicolumn{2}{|l|}{$\mathrm{SE}$} \\
\hline & & $\geq 26 \mathrm{~mm}^{*}$ & $<26 \mathrm{~mm}$ & $\leq-6 D^{*}$ & $>-6 \mathrm{D}$ \\
\hline$n$ & 33 & 7 & 26 & 9 & 24 \\
\hline Before correction, $\mathrm{mm}^{2}$ & $0.26 \pm 0.10$ & $0.24 \pm 0.09$ & $0.27 \pm 0.11$ & $0.24 \pm 0.07$ & $0.26 \pm 0.11$ \\
\hline After correction, $\mathrm{mm}^{2}$ & $0.27 \pm 0.10$ & $0.27 \pm 0.10$ & $0.26 \pm 0.10$ & $0.27 \pm 0.07$ & $0.26 \pm 0.11$ \\
\hline
\end{tabular}

The data represent the mean $\pm \mathrm{SD}$. ${ }^{*} p<0.01$, paired $t$ test. FAZ, foveal avascular zone; SE, spherical equivalent.

The FAZ areas before and after correction of the axial length or SE were compared using paired $t$ test. Correlation between changes in the FAZ area before and after the correction of ocular axial length and axial length was examined using Pearson's correlation coefficient. Correlation between change in FAZ area before and after correction of SE and SE value was examined using Pearson's correlation coefficient. We performed subgroup analysis by classifying data into two groups of myopic and non-myopic eyes. We set the axial length to 26.0 $\mathrm{mm}$ or SE value to -6.0 diopters (D) because high myopia was defined as an SE >-6 D or an axial length greater than or equal to $26 \mathrm{~mm}$ by Flores-Moreno et al. [18], who investigated a relationship between axial length and choroidal thickness in eyes with high myopia. All data analyses were performed using JMP (SAS Institute Inc., Cary, NC, USA) and SPSS statistical software (SPSS Inc., Chicago, IL, USA), and statistical significance was defined as $p<0.05$.

\section{Results}

The right eyes of 33 healthy subjects were enrolled and all subjects were eligible for the study. The average age of participants was $21.9 \pm 0.6$ years, of which 22 were female subjects and 11 were male subjects. Table 1 summarizes the subject baseline demographics and clinical characteristics. The mean axial length was $24.87 \pm 1.17 \mathrm{~mm}$ and mean SE value was $-3.64 \pm 2.83 \mathrm{D}$.

The inter-scan reproducibility for manual FAZ measurement was accurate both before and after axial length correction (CV 4.2\% [2.7-5.8], ICC 0.98 [0.95-0.99] before correction, and CV 4.5\% [2.8-6.1], ICC 0.97 [0.95-0.99] after correction). The FAZ area was $0.26 \pm 0.10$ $\mathrm{mm}^{2}$ before the axial length correction and $0.27 \pm 0.10 \mathrm{~mm}^{2}$ after the correction. The FAZ area showed no correctional difference in eyes for which the axial length was shorter than 26.0 $\mathrm{mm}$ or SE value was more than $-6.0 \mathrm{D}$. Eyes for which the axial length was longer than or equal to $26.0 \mathrm{~mm}$ or the SE value was less than or equal to $-6.0 \mathrm{D}$ showed that the FAZ area after correction was significantly larger than before correction $(p<0.01$; Table 2$)$.

A significant positive correlation between the change of FAZ area after correction and the axial length was observed $\left(R^{2}=0.88, p<0.01 ;\right.$ Fig. 2 a). A significant negative correlation between the change of FAZ area after correction and the SE was also found $\left(R^{2}=0.55, p<0.01\right.$; Fig. $\left.2 \mathrm{~b}\right)$. 
Fig. 2. a Correlation between change of FAZ area (before and after correction) and axial length. There was significant positive correlation between the change of FAZ area and axial length $\left(R^{2}=\right.$ $0.88, p<0.01)$. The regression line indicates no change between FAZ area before and after axial length correction when the axial length was approximately 24.6 $\mathrm{mm}$. The 95\% confidential interval is indicated with a blue band. b Correlation between the change of FAZ area (before and after correction) and SE. There was significant negative correlation between the change of FAZ area and axial length $\left(R^{2}=0.88, p<0.01\right)$. The regression line indicates no change between FAZ area before and after axial length correction when the SE was approximately -3.0 D. The $95 \%$ confidential interval is indicated with a blue band.

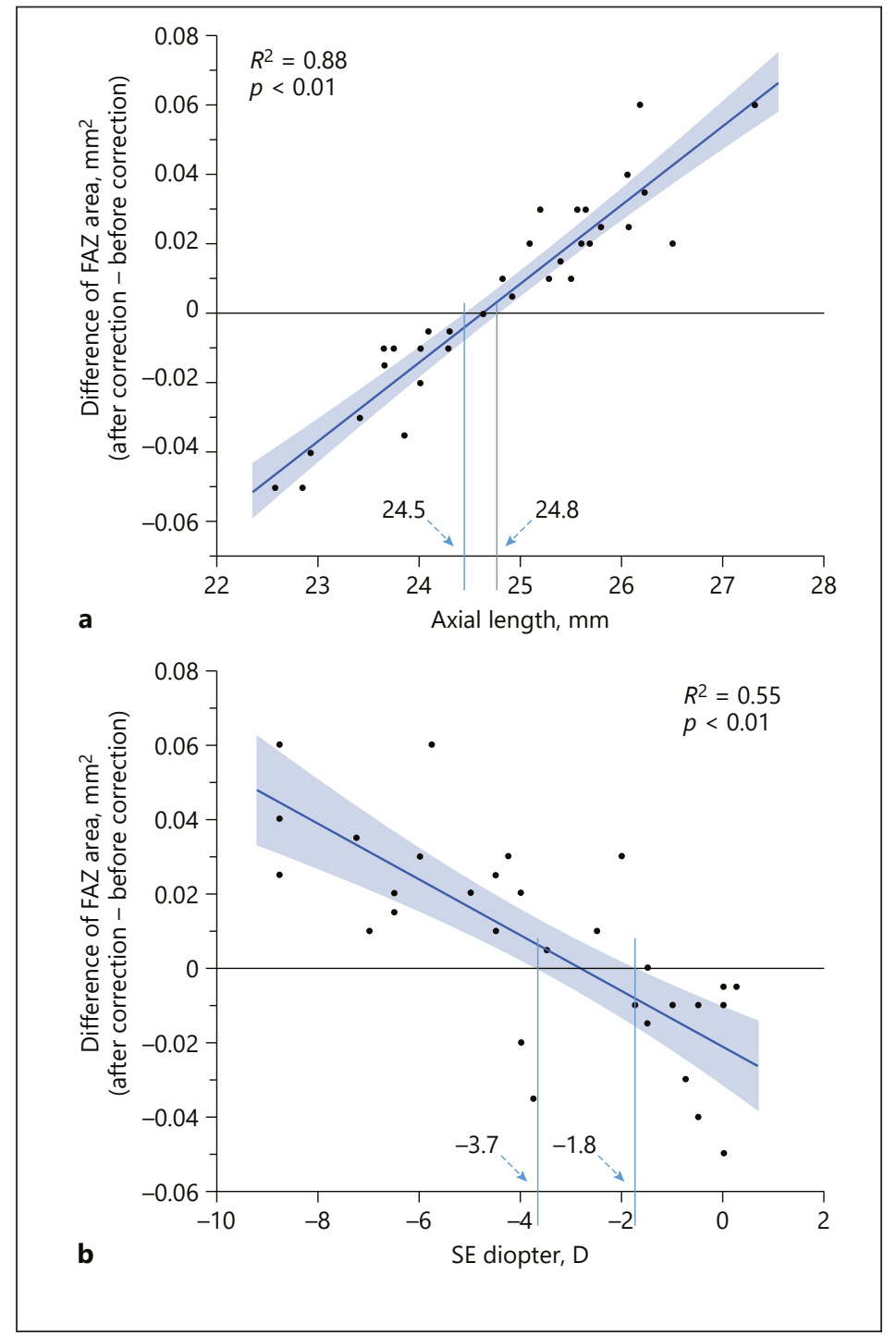

\section{Discussion}

In this study, we measured the FAZ area in healthy controls, investigating the magnification effect on area by means of axial length or SE correction. Linderman et al. [14] reported that the average error of the FAZ area is $8.29 \%$, with the maximum error being $31.36 \%$ by axis correction. The value of the FAZ area measured using SD-OCTA RS-3000 Advance was comparable to a previous report [19]. The FAZ area was not significantly changed by axial length or SE correction, but for the eyes with severe myopia, the FAZ area after axial length or SE correction was significantly larger than before correction. It was also found that the amount of change in the FAZ area had a positive correlation between the axial lengths and a negative correlation between the refraction values.

Shahlaee et al. [20] and Tan et al. [21] reported that the mean FAZ area was $0.27 \pm 0.10$ $\mathrm{mm}^{2}$ using RTVue XR Avanti (Optovue) and $0.24 \mathrm{~mm}^{2}$ using AngioVue (Optovue), respectively. Iafe et al. [10] reported that the mean FAZ area was $0.29 \pm 0.11 \mathrm{~mm}^{2}$ when measured with RTVue XR Avanti, and age was a predictor of FAZ area for subjects aged between 20 and 29 years. In the current study, the mean FAZ area measured by RS-3000 Advance (NIDEK) 
was $0.26 \pm 0.10$ and $0.27 \pm 0.10 \mathrm{~mm}^{2}$ before and after axial length correction, respectively. These values were similar to those in previous reports.

The previous reports $[17,19,22]$ showed good reproducibility of vessel density measurement and FAZ measurement for macula scan using RS-3000 Advance. Similarly, the inter-scan reproducibility was good in the current study. The values of the FAZ area measured with RS-3000 Advance were suitable for analysis. In the current study, the difference of FAZ area before and after axial correction was positively correlated with axial length and negatively correlated with SE value. Therefore, without axial length correction, the FAZ area is overestimated in the eye with a shorter axial length and underestimated in the eye with an axis longer than $24.6 \mathrm{~mm}$ (Fig. 2a).

In the high myopic eyes, in which the axial length was more than $26.0 \mathrm{~mm}$ or SE was lower than $-6 \mathrm{D}$, the mean FAZ area after correction was significantly higher than before correction $(p<0.01)$. On the contrary, in eyes without high myopia, there was no difference in the FAZ area between before- and after-correction values. This may be due to the limited number of subjects in the current study whose axial length was less than $24.6 \mathrm{~mm}$ (13 eyes, 39\%).

Of utmost importance to our study, there was linear correlation between the shift of FAZ area after correction and either axial length or SE. This means the greater the distance of axial length from $24.5 \mathrm{~mm}$, the smaller the SE from -3.0 D, and the more the FAZ area was underestimated. For example, in an eye with an axial length of $27 \mathrm{~mm}$, the FAZ area would be estimated as much as approximately $25 \%$ smaller without correction. Similarly, the degree to which the axial length was shorter than $24.5 \mathrm{~mm}$, or the greater the SE value than $-3.0 \mathrm{D}$, the more the FAZ area would be overestimated.

Although there have already been several reports that examined the FAZ area in various retinal diseases and glaucoma [11-13, 23, 24], the magnification effect was not always considered in the evaluation on the FAZ area. Linderman et al. [14] reported that both axial length and refraction abnormality influence the measurement results due to the magnification effect. Our results and their report show the importance of considering the magnification effect for evaluating FAZ area.

Failing to correct the axial length or refractive error would result in abnormal measurements, and therefore the FAZ area would be incorrectly evaluated. Thus, one should consider magnification effect on the evaluation of the FAZ area, especially in eyes with severe myopia.

Our study had several limitations. First, the sample size was small and included only young subjects. Further studies with larger sample sizes and various age groups are needed. Second, the number of females was greater than males. Tan et al. [21] reported that the FAZ area is significantly larger in females than in males. Further studies adjusting for sex or separately evaluating females and males are needed. Third, we measured FAZ area only by a manual and single device. There are several methods to measure FAZ area (e.g., manual, semi-automatic, automatic) [25]. The FAZ area varied depending on the measurement method and instrument $[8,19,26,27]$. Although each OCTA imaging system has been reported to provide highly reproducible FAZ images with a high inter-operator concordance level $[25,28]$, the method of quantifying OCTA images and the method of correcting magnification effects has not been standardized and there is no compatibility between each device $[26,28]$. Linderman et al. [14] reported that the FAZ area is larger when measuring manually rather than by automatic or semi-automatic methods. In addition, previous reports $[8,20$, 26 , 27] state that even with the same device, the value of FAZ area varied depending on the measuring method.

Therefore, when comparing the FAZ area among different facilities or different OCTA devices, multiple variables must be considered, including magnification effect, measuring method, OCTA devices, and sex. 


\section{Conclusion}

The value of the FAZ area measured using SD-OCTA RS-3000 Advance was comparable to previous reports. The FAZ area was not significantly changed before or after the correction of axial length or SE when all cases were analyzed together. However, the FAZ area was significantly altered after correction with axial length or refractive error in myopic eyes. Furthermore, the difference of FAZ area before and after correction shows significant correlation between axial length and SE. Especially in myopic eyes, magnification effect should be considered when evaluating the FAZ area with axial length or refractive error.

\section{Acknowledgements}

We would like to thank Editage (www.editage.com) for English language editing.

\section{Statement of Ethics}

This prospective study was approved by the Ethics Committee of Teikyo University School of Medicine (study ID No. 15-228). All studies conducted adhered to the tenets of the Declaration of Helsinki, and written informed consent was obtained from each participant before any study examination was performed. The participant anonymity is preserved.

\section{Disclosure Statement}

None of the authors have any financial disclosures.

\section{Funding Sources}

This study was supported in part by the Japan Society for the Promotion of Science (JSPS; KAKENHI grant No. 17K11430).

\section{Author Contributions}

K.S., G.T., and S.M. designed the study and M.S. collected the data. M.S. and Y.Y. performed the analysis and prepared the figures and tables. M.S. and Y.Y. prepared a draft and Y.Y., T.S., K.S., A.M., and Y.K. gave critical suggestions. Y.Y., T.S., and K.S. revised the manuscript and finalized it. All authors agree to be accountable for all aspects of the work. All authors attest that they meet the current ICMJE criteria for authorship.

\section{References}

1 Chui TY, VanNasdale DA, Elsner AE, Burns SA. The association between the foveal avascular zone and retinal thickness. Invest Ophthalmol Vis Sci. 2014 Sep;55(10):6870-7.

2 Conrath J, Giorgi R, Raccah D, Ridings B. Foveal avascular zone in diabetic retinopathy: quantitative vs qualitative assessment. Eye (Lond). 2005 Mar;19(3):322-6.

3 Sander B, Larsen M, Engler C, Lund-Andersen H. Absence of foveal avascular zone demonstrated by laser scanning fluorescein angiography. Acta Ophthalmol (Copenh). 1994 Oct;72(5):550-2. 
4 Yoon YS, Woo JM, Woo JE, Min JK. Superficial foveal avascular zone area changes before and after idiopathic epiretinal membrane surgery. Int J Ophthalmol. 2018 Oct;11(10):1711-5.

5 Henke S, Papapostolou I, Heimes B, Lommatzsch A, Pauleikhoff D, Spital G. OCT-Angiography in diabetic maculopathy : comparison between microaneurysms and the foveal avascular zone with flourescein angiography. Ophthalmologe. 2018 Nov;115(11):941-7. German.

6 Tang FY, Ng DS, Lam A, Luk F, Wong R, Chan C, et al. Determinants of quantitative optical coherence tomography angiography metrics in patients with diabetes. Sci Rep. 2017 May;7(1):2575.

7 Koulisis N, Kim AY, Chu Z, Shahidzadeh A, Burkemper B, Olmos de Koo LC, et al. Quantitative microvascular analysis of retinal venous occlusions by spectral domain optical coherence tomography angiography. PLoS One. 2017 Apr;12(4):e0176404.

8 Kita Y, Inoue M, Kita R, Sano M, Orihara T, Itoh Y, et al. Changes in the size of the foveal avascular zone after vitrectomy with internal limiting membrane peeling for a macular hole. Jpn J Ophthalmol. 2017 Nov;61(6):465-71.

9 Nelis P, Alten F, Clemens CR, Heiduschka P, Eter N. Quantification of changes in foveal capillary architecture caused by idiopathic epiretinal membrane using OCT angiography. Graefes Arch Clin Exp Ophthalmol. 2017 Jul;255(7):1319-24.

10 Iafe NA, Phasukkijwatana N, Chen X, Sarraf D. Retinal capillary density and foveal avascular zone area are agedependent: quantitative analysis using optical coherence tomography angiography. Invest Ophthalmol Vis Sci. 2016 Oct;57(13):5780-7.

11 Yoshikawa Y, Shoji T, Kanno J, Ibuki H, Ozaki K, Ishii H, et al. Evaluation of microvascular changes in the macular area of eyes with rhegmatogenous retinal detachment without macular involvement using sweptsource optical coherence tomography angiography. Clin Ophthalmol. 2018 Oct;12:2059-67.

12 Zivkovic M, Dayanir V, Kocaturk T, Zlatanovic M, Zlatanovic G, Jaksic V, et al. Foveal avascular zone in normal tension glaucoma measured by optical coherence tomography angiography. BioMed Res Int. 2017;2017: 3079141.

13 Mintz-Hittner HA, Knight-Nanan DM, Satriano DR, Kretzer FL. A small foveal avascular zone may be an historic mark of prematurity. Ophthalmology. 1999 Jul;106(7):1409-13.

14 Linderman R, Salmon AE, Strampe M, Russillo M, Khan J, Carroll J. Assessing the accuracy of foveal avascular zone measurements using optical coherence tomography angiography: segmentation and scaling. Transl Vis Sci Technol. 2017 Jun;6(3):16.

15 Morgan IG, Ohno-Matsui K, Saw SM. Myopia. Lancet. 2012 May;379(9827):1739-48.

16 Sampson DM, Gong P, An D, Menghini M, Hansen A, Mackey DA, et al. Axial length variation impacts on superficial retinal vessel density and foveal avascular zone area measurements using optical coherence tomography angiography. Invest Ophthalmol Vis Sci. 2017 Jun;58(7):3065-72.

17 Al-Sheikh M, Tepelus TC, Nazikyan T, Sadda SR. Repeatability of automated vessel density measurements using optical coherence tomography angiography. Br J Ophthalmol. 2017 Apr;101(4):449-52.

18 Flores-Moreno I, Lugo F, Duker JS, Ruiz-Moreno JM. The relationship between axial length and choroidal thickness in eyes with high myopia. Am J Ophthalmol. 2013 Feb;155(2):314-319.e1.

19 Shiihara H, Sakamoto T, Yamashita T, Kakiuchi N, Otsuka H, Terasaki H, et al. Reproducibility and differences in area of foveal avascular zone measured by three different optical coherence tomographic angiography instruments. Sci Rep. 2017 Aug;7(1):9853.

20 Shahlaee A, Pefkianaki M, Hsu J, Ho AC. Measurement of foveal avascular zone dimensions and its reliability in healthy eyes using optical coherence tomography angiography. Am J Ophthalmol. 2016;161:50-55.e51.

21 Tan CS, Lim LW, Chow VS, Chay IW, Tan S, Cheong KX, et al. Optical coherence tomography angiography evaluation of the parafoveal vasculature and its relationship with ocular factors. Invest Ophthalmol Vis Sci. 2016 Jul;57(9):OCT224-34.

22 Pilotto E, Frizziero L, Crepaldi A, Della Dora E, Deganello D, Longhin E, et al. Repeatability and reproducibility of foveal avascular zone area measurement on normal eyes by different optical coherence tomography angiography instruments. Ophthalmic Res. 2018;59(4):206-11.

23 Balaratnasingam C, Inoue M, Ahn S, McCann J, Dhrami-Gavazi E, Yannuzzi LA, et al. Visual acuity is correlated with the area of the foveal avascular zone in diabetic retinopathy and retinal vein occlusion. Ophthalmology. 2016 Nov; 123(11):2352-67.

24 Jauregui R, Park KS, Duong JK, Mahajan VB, Tsang SH. Quantitative progression of retinitis pigmentosa by optical coherence tomography angiography. Sci Rep. 2018 Sep;8(1):13130.

25 La Spina C, Carnevali A, Marchese A, Querques G, Bandello F. Reproducibility and reliability of optical coherence tomography angiography for foveal avascular zone evaluation and measurement in different settings. Retina. 2017 Sep;37(9):1636-41.

26 Magrath GN, Say EA, Sioufi K, Ferenczy S, Samara WA, Shields CL. Variability in foveal avascular zone and capillary density using optical coherence tomography angiography machines in healthy eyes. Retina. 2017 Nov;37(11):2102-11.

27 Rabiolo A, Gelormini F, Marchese A, Cicinelli MV, Triolo G, Sacconi R, et al. Macular perfusion parameters in different angiocube sizes: does the size matter in quantitative optical coherence tomography angiography? Invest Ophthalmol Vis Sci. 2018 Jan;59(1):231-7.

28 Shoji T, Yoshikawa Y, Kanno J, Ishii H, Ibuki H, Ozaki K, et al. Reproducibility of macular vessel density calculations via imaging with two different swept-source optical coherence tomography angiography systems. Transl Vis Sci Technol. 2018 Dec;7(6):31. 